\title{
Análisis de los factores que componen un sistema de gestión empresarial: estudio de caso para la Dirección de Vivienda
}

\section{Analysis of the factors making up a business management system: case study for the Housing Directorate}

Dr. Gustavo Adolfo Rubio Rodríguez Universidad Cooperativa de Colombia, Colombia

gustavo.rubio@campusucc.edu.co

\author{
Mag. Carlos Arturo Téllez Bedoya \\ Universidad San Buenaventura Bogotá, \\ Colombia \\ ctellez@usbbog.edu.co
}

\author{
Mag. Dustin Tahisin Gómez Rodríguez \\ Universitaria Agustiniana Bogotá, \\ Colombia \\ dustin.gomez@uniagustiniana.edu.co
}

\section{RESUMEN:}

El objetivo del presente artículo fue analizar los factores que integran un sistema de gestión empresarial que permita optimizar la administración de los recursos humanos, materiales y financieros, de los cuales las organizaciones disponen para el cumplimiento de sus actividades. La metodología es de corte cuantitativo y el método de estudio fue el de caso por medio del análisis de información a través de instrumentos como el software SPSS, así como el empleo de diferentes etapas, permitiendo la realización del trabajo de campo, pues el tipo de investigación es descriptivo. La principal conclusión es que los resultados del estudio adelantado no fueron alentadores, se evidencia que hoy no existe homogeneidad en las variables analizadas y ello manifiesta las falencias contenidas al interior de la empresa objeto de estudio.

\section{ABSTRACT:}

The objective of this article was to analyze the factors that make up a business management system, which enables to optimize the administration of human, material and financial resources at the disposal of organizations so that they can perform their activities. This essay is derived from a process, whose theoretical foundation is set into the conceptualization and contextualization of the management system, in the stages covered by the said system via proper use. The methodology is quantitative and the study method was centered on a case by means of information analysis through instruments such as SPSS software. Also, different stages were used, allowing field work to be carried out, as the type of research is descriptive. The main conclusion is that the results obtained in the advanced study were not encouraging. It is evident that today there is no homogeneity in the analyzed variables and this reveals the flaws contained within the company under study.

\section{KEYWORDS}

MANAGEMENT SYSTEM, ADMINISTRATION, PLANNING, ADMINISTRATIVE PROCESS

\section{PALAVRAS CHAVES \\ SISTEMA DE GESTÃO, ADMINISTRAÇÃO, PLANEJAMENTO, PROCESSO \\ ADMINISTRATIVO.}

O objetivo do presente artigo foi analisar os fatores que compõem um sistema de gestão empresarial que otimiza a administração de recursos humanos, materiais e financeiros, dos quais as organizações dispõem para o cumprimento de suas atividades. Este trabalho conceituação e na contextualização do sistema de gestão, nas etapas é quantitativa e o método de estudo foi o caso por meio da análise de informações por meio de instrumentos como o software SPSS o diferentes etapas, permitindo a realização do trabalho de campo, já que o tipo de investigação é descritiva. A conclusão é que os resultados do estudo avançado não foram animadores, é evidente que hoje não há homogeneidade nas interior da empresa objeto de estudo.

\section{RÉSUMÉ:}

L'objectif de cet article a été d'analyser les facteurs constituant un système de gestion d'entreprise qui permet d'optimiser l'administration des ressources humaines, matérielles et financières à disposition des organisations pour effectuer leurs activités. Cet essai découle d'un processus, dont le fondement théorique est inscrit dans la conceptualisation et contextualisation du système de gestion, dans les étapes couvertes par ce système et son utilisation adéquate. La méthodologie est de type quantitatif et la méthode d'étude est basée sur un cas au moyen de l'analyse d'information via des instruments tel que le logiciel SPSS, ainsi que par l'emploi de différentes étapes permettant la réalisation du travail de terrain, étant donné que la recherche est descriptive. La conclusion principale est que les résultats de l'étude avancée n'ont pas été encourageants. Il apparaît qu'aujourd'hui il n'y a pas d'homogénéité dans les variables analysées et cela révèle les manquements au sein de l'entreprise faisant objet d'étude.
ADMINISTRACIÓN, PLANEACIÓN, PROCESO ADMINISTRATIVO.

\section{MOTS CLÉS}

SYSTEME DE GESTION, ADMINISTRATION, PLANIFICATION, PROCESSUS ADMINISTRATIF. 


\section{INTRODUCCIÓN}

Los cambios organizacionales son fenómenos que permean las decisiones de los gestores de las firmas, así como de la dirección y la administración de empresas (Gómez y Rincón, 2016). Decisivamente, las organizaciones deben adaptar sus desempeños, sus estrategias, su gestión y su estructura a las dinámicas de su entorno inmediato en primera instancia. Sin olvidar sus relaciones tanto nacionales como internacionales, en virtud de las fluctuaciones del mercado (Katz y Kahn, 1978).

En efecto, el cambio tecnológico, los tratados de libre comercio, la competitividad incesante del capital social, humano e intelectual, así como del intangible, la destrucción y creación de empresas (Schumpeter, 1967), la adquisición de empresas por inversionistas extranjeros (Contreras y Barbosa, 2013), la innovación y la integración empresarial (Daft, 2007; Mintzberge, 1984; Poole y Van de Ven, 2004), el aprendizaje organizacional (Ahumada, 2001; Nonaka, Takeuchi y Umemoto, 1996), las reconfiguraciones de tejido social -desde la perspectiva de ciencia política- frente a las comunidades, la incorporación en costos dados los impactos al medio ambiente, las diferentes formas de mercadeo al retail o al detal (Rincón, Niño y Gómez, 2017; Gómez, Rincón y Niño, 2017) y la competitividad (Porter, 1998), son variables o entramados que posicionan o eliminan la gestión, la organización y la valoración de las empresas. Por consiguiente, es imperativo evidenciar cómo las organizaciones cultivan estas dinámicas, así como las diferentes formas de interactuar con el mercado y adaptarse, dado que este es cada vez más competitivo en aras de la globalización que exhiben algunos países y organizaciones.

En consecuencia, el sector privado representa hoy un actor dominante en los mercados y, derivado de ello, debe implementar en su gestión prácticas diferenciadas del empirismo, que conlleven tanto el diagnóstico como la solución de problemáticas internas, a la vez que propicien un adecuado relacionamiento con los actores inmersos en el tejido social y la creación de lazos de confianza con los propietarios, colaboradores, clientes y proveedores (Losada, Bonilla y Gómez, 2018). Igualmente, este tipo de acciones realizadas en los diversos niveles de dirección de las organizaciones han denotado que, "las buenas prácticas gerenciales, entre ellas los mecanismos que poseen para la transferencia del conocimiento al interior de la empresa, combinadas con los recursos y las oportunidades disponibles, son las razones que los empresarios aducen para haber logrado mantener una ventaja competitiva" (Franco y Urbano, 2010, p. 91).

Lo anterior, sin duda alguna, impacta positivamente en el rol que las organizaciones poseen en la sociedad, a la vez que propugnan por el avance continuo en la búsqueda de un mejor posicionamiento, así como el de sus productos y servicios. Ello, a futuro, redunda en el mejoramiento de indicadores internos y externos, al igual que en un mayor crecimiento, estabilidad y rentabilidad del negocio.

Teniendo en cuenta lo anterior, y a partir de la oportunidad que se presenta en constituir una alternativa de solución propicia a las dificultades de gestión que actualmente enfrentan algunas organizaciones en el Estado colombiano, este estudio diagnóstico de la situación actual de ellas busca identificar las causas que están originando dichas problemáticas. Además, se emplearon los sistemas de gestión como aspectos centrales de la investigación, al considerarlos como instrumentos que coadyuvan en la organización de los procesos al interior de las áreas funcionales y, en este sentido, promover el cumplimento de cada uno de los elementos que componen el proceso administrativo.

los resultados evidencian que los factores de mayor incidencia en el fracaso de los emprendimientos son, por orden jerárquico: los elementos financieros, organizacionales y/o administrativos y el mercadeo. Los emprendedores coincidieron en que no contaban con herramientas adecuadas a nivel financiero y organizacional al momento de desarrollar y consolidar sus negocios (...) En cuanto a la parte organizacional y/o administrativa, se destacan problemas en la planeación y la falta de indicadores y métodos de gestión que permitan realizar un seguimiento riguroso de las tendencias del negocio (Pardo y Alfonso, 2015, p. 67). 
A partir de la incidencia que significa para las organizaciones el desarrollo de instrumentos que faciliten su gestión, a la vez que existe un importante número de empresas que cada año deben concluir sus operaciones por este tipo de situaciones, se busca a continuación analizar la aplicación de los principales factores inmersos en los sistemas de gestión en organizaciones del país, teniendo en cuenta un ejercicio de estudio de caso aplicado al sector vivienda. Para cumplir con esta finalidad, el documento contiene tres apartados, cuyo contenido precisa la siguiente información:

Inicialmente, se exponen los antecedentes del proyecto; estos se presentan a manera de fundamentación teórica, a fin de soportar empíricamente el estudio. Para mayor exactitud en el entendimiento del trabajo, se relacionan algunos conceptos de sistema de gestión, así como las etapas que surten en este y el uso con el cual se debe adoptar. Luego, se relaciona el diagnóstico de la entidad seleccionada como estudio de caso, la cual tiene como objeto social direccionar planes de vivienda; dicho apartado comprende la metodología e instrumentos empleados. De igual forma, se presentan los resultados obtenidos en el trabajo de campo y la discusión de estos. El propósito que se persigue en este apartado es evidenciar la existencia del problema a partir de los datos conseguidos.

Por último, el trabajo desarrollado vislumbró un acierto en la organización seleccionada como objeto de estudio, ya que, una vez se evalúen y diseñen los parámetros a tener en cuenta en la elaboración del sistema de gestión, al interior de la empresa se podrá controlar en mayor medida el proceso de asignación de viviendas, dar fomento al uso apropiado de los recursos, efectuar los cobros de forma organizada, recolectar información a través de encuestas con las cuales se conozca el nivel de satisfacción de colaboradores y clientes, hacer un seguimiento pertinente a los puestos de trabajo asignados por la empresa y automatizar los procesos administrativos con el fin de optimizar su funcionamiento.

\section{FUNDAMENTACIÓN TEÓRICA}

Teniendo en cuenta el concepto de sistema de gestión que ha venido evolucionando en el contexto de la ciencia administrativa, el cual se relaciona directamente con el proceso administrativo, se presentan a continuación las principales nociones teóricas que tuvo en cuenta este trabajo investigativo, de forma previa al análisis que se llevó a cabo en el estudio de caso objeto de estudio.

\section{SISTEMA DE GESTIÓN}

El proceso administrativo surgió en la propuesta planteada por Fayol al interior de la teoría clásica, buscando aproximarse a la comprensión de la labor de gestión en las organizaciones. Años más tarde, la teoría neoclásica retoma y actualiza dichos componentes del proceso administrativo y hoy se presentan de manera aplicada en los sistemas de gestión.

Es de resaltar que, al interior del concepto enunciado, sobresale la necesidad de continuidad en sus etapas, así como el compromiso en aras de impactar positivamente la efectividad de las empresas. Principalmente, la literatura académica reconoce la existencia de cuatro pasos que componen estos sistemas de gestión y que se repiten de forma recurrente, de tal manera que se obtenga una mejora al final del ciclo. Lo anterior se evidencia en la propuesta de Téllez (2015), en la cual se enuncia que un sistema de gestión se describe como una herramienta con atributos para que cualquier organización planee, organice, dirija y controle los diversos procesos, en aras de dar cumplimiento a la misión, visión y objetivos de la empresa, de la mano con altos estándares de calidad, cuya medición se efectúa a partir de los indicadores de satisfacción de los clientes.

Por otro lado, Fraguela, Carral, Iglesias, Castro y Rodríguez (2011) definen un sistema de gestión como un conjunto de aspectos relacionados entre sí, los cuales interactúan continuamente y que impactan en el planteamiento de las 
políticas, estrategias y el cumplimiento de los objetivos. Cabe mencionar, independientemente del autor que se analice, que resaltan los siguientes conceptos: planeación, organización, documentación, formación y cualificación, control, implementación, evaluación, mejora y comunicación. De forma previa al abordaje de dichas nociones, es reconocido que los sistemas de gestión buscan incidir en la reducción de costos, la agilización de los procesos, la disminución de los trámites y la burocracia, el desarrollo de una óptica dirigida a los negocios y la motivación de los colaboradores. Lo anterior, sin duda alguna, contribuye a que el administrador tenga una visión holística de la organización, así como de los actores sociales con los cuales interactúa de manera permanente en el mercado.

\section{ETAPAS DEL SISTEMA DE GESTIÓN}

La composición de los sistemas de gestión mediante la implementación de las siguientes etapas: ideación, planeación, implementación y control. Cabe destacar que esta clasificación guarda una secuencia lógica y ordenada, tal como se ilustra en la figura 1; es decir, la identificación de un proceso o etapa no se puede adelantar sin tener en cuenta la ocurrencia del que lo precede.

\section{IDEACIÓN}

El propósito de esta etapa es ocuparse de la idea que servirá de guía a los primeros pasos del proceso con el que se aspira diseñar el sistema de gestión. Actualmente, subsisten varias metodologías para lograr depurar la idea. Entre ellas, sobresalen técnicas como brainstorming, 6.3.5, la ley de la C, ojos limpios, flor de loto y el catálogo.

La más reconocida es brainstorming; surge de la propuesta de Alex Osborn y busca alentar la generación de ideas en forma libre y espontánea; para ello, se recomienda que se reúna un grupo de diez a catorce personas, donde, tras la descripción de un problema, se precise el surgimiento de alternativas de solución (Fernández, 2005).

Una vez que se ha aplicado la técnica de generación de ideas, se recomienda que se tome nota en un tablero y se agrupen las ideas semejantes, con colaboración de los asistentes. Posterior a este momento, se puede llevar a cabo la aplicación de técnicas que permiten evaluar las ideas, seleccionar las más relevantes y evaluar las posibles contingencias que se desarrollen en la implementación de dichas ideas.

\section{PLANEACIÓN}

De acuerdo con Baena (2015), la planeación es la función que tiene como objetivo fijar el curso concreto de acción que ha de seguirse, estableciendo los principios que habrán de orientar los sistemas de gestión, la secuencia de operaciones para realizarlo y las determinaciones de recursos necesarios para su realización. La planeación también implica la tarea de definir los objetivos estratégicos, tácticos y operativos, buscando el alcance de las estrategias y llevando a cabo estas a través de tácticas, directrices y normas que estén en coherencia con la jerarquía completa de planes de la organización. En esta etapa, se comprende a su vez el desarrollo de la función de organización, inmersa en el proceso administrativo, es decir, el diseño de la estructura material y social, aclarando los colaboradores asignados, sus roles y los niveles de responsabilidad y autoridad que deben cumplir en la organización.

Cabe anotar que los elementos que componen la formulación de las tres estrategias deben ser objeto de implementación en cualquier estructura organizacional, independientemente de la naturaleza y del tamaño de la organización, debido a que su afectación con respecto a los resultados esperados dependerá de estos factores. En otras palabras, una empresa cuya estructura guarde características complejas, reflejará resultados operativos y demás aspectos de acuerdo con dicha complejidad. En igual medida, sucederá con aquellas organizaciones cuya composición operativa sea mediana o pequeña.

La planeación comprende a su vez diversas etapas, las cuales promueven su dinamismo, flexibilidad y continuidad. Generalmente, la literatura académica ha identificado rasgos comunes en dichas etapas, incluyendo así el análisis 
externo (de oportunidades y amenazas), el análisis interno (de fortalezas y debilidades) y, derivado de ello, la identificación de una estrategia competitiva, la cual incidirá en la proposición de objetivos, metas y otros tipos de elementos.

En el ámbito de los negocios y a nivel funcional, los resultados de la planeación se enmarcan en propuestas de programas estratégicos de acción y programación de instrumentos, como los presupuestos. Estas propuestas son, finalmente, evaluadas y consolidadas a nivel corporativo.

\section{IMPLEMENTACIÓN}

Generalmente, esta fase se relaciona con el concepto de gestión y ello se presupone como una acción desarrollada por la alta dirección que se enmarca en el desarrollo de la ciencia administrativa. Chiavenato (2002) define este concepto como un conjunto de políticas, nociones y prácticas que guardan coherencia y que pretenden alcanzar los objetivos de la organización en forma efectiva. En un contexto empresarial, ello se refiere a la puesta en marcha de las habilidades directivas, como la toma de decisiones, la comunicación, la motivación y la solución de conflictos. En efecto, cabe mencionar que las decisiones y posteriores acciones que se toman en cumplimiento de un plan se basan en herramientas, las cuales están sistémicamente relacionadas y que se obtienen del adecuado desarrollo en el proceso de planeación.

\section{CONTROL}

Esta última etapa de los sistemas de gestión y que, en general, se comprende en el proceso administrativo, se define como una función administrativa que posee esencialmente un rol de regulación, verificando que los criterios seleccionados inicialmente se lleven a la práctica. La principal finalidad del control es el diagnóstico de errores, la identificación de variaciones y la prevención y corrección de las desviaciones identificadas. Por ende, el control debe estar relacionado con los planes inicialmente definidos, debe permitir la medición y cuantificación de los resultados, la detección de desviaciones y el establecimiento de medidas correctivas y preventivas.

\section{ETAPAS DEL CONTROL}

Chiavenato (2002) menciona que el proceso de control se entiende como cíclico, basado en los siguientes pasos:

- Establecimiento de estándares y criterios, es decir, enunciar las disposiciones establecidas para observar lo que debe hacerse y cuál es el desempeño o resultado esperado.

- Observación del desempeño, verificación de los resultados obtenidos sobre la operación analizada.

- Comparación del desempeño con el estándar establecido, determinar los límites de la variación en la operación para observar qué es lo normal o aceptable y qué no lo es.

- Acción correctiva, establecida para mantener las operaciones en los estándares y corregir el desvío entre el desempeño real y el desempeño esperado.

\section{USOS DE SISTEMA DE GESTIÓN}

La empresa que quiera sostenerse en el mercado tendrá que adaptarse a las exigencias de este, implementando sistemas de gestión que integren sus productos y servicios, con elementos que generen confianza y beneficien la decisión de compra por parte de los clientes y demás actores sociales del entorno (Fraguela, Carral, Iglesias, Castro y Rodríguez, 2011). A continuación, se exponen dos trabajos en los cuales se han implementado sistemas de gestión 
como herramientas administrativas que pueden ser aplicadas al interior de una organización y que contribuyen al mejoramiento de su comportamiento en el modelo empresarial, así como al mantenimiento o alcance de un mayor posicionamiento.

El primer trabajo se relaciona con el diseño e implementación de un sistema de gestión de calidad en una universidad y el segundo con el diseño de un sistema de gestión para una asociación. Ambos trabajos tienen algo en común: al igual que todas las organizaciones, les asiste el interés por desarrollarse en todos sus procesos, ser conscientes de la necesidad de emplear un sistema de gestión que permita controlar la operatividad de la empresa, con la participación de todos los colaboradores, y con el propósito de conquistar los corolarios pronosticados.

Entre las recomendaciones presentadas para la aplicación de un modelo de gestión en un programa académico de una universidad, Inche, Chung y Salas (2010) recomiendan que el personal sea el elemento más importante en un sistema de gestión; por ende, la satisfacción de los colaboradores debe ocupar en la mente de los propietarios, administrativos y demás actores sociales una posición predominante. El ambiente en el que el colaborador desarrolla su función laboral contribuirá, en consecuencia, a generar en él un alto sentido de pertenencia a la empresa a la que está vinculado. Por lo tanto, el empresario debe concentrar ingentes esfuerzos que redunden en el personal que tiene a cargo.

Por otro lado, en el estudio denominado "Diseño de un sistema de gestión administrativo para la asociación Asomujeres Proactivas en el municipio de Pereira Risaralda”, de Rengifo y Ramírez (2010), se concluye que las entidades sin ánimo de lucro deben tomar consciencia de la necesidad de tramitar desde una configuración administrativa y estratégica, hasta la ejecución de un sistema de gestión en su modelo empresarial. Esta conclusión evidencia que no solo las organizaciones que forman parte de la economía civil pueden ser objeto de la implementación de este tipo de procesos, sino también las empresas que componen la economía social, pues tanto unas como otras propugnan por mejorar su funcionamiento organizacional e institucional.

En el ámbito regional, especialmente en Latinoamérica, se han llevado a cabo diferentes programas mediante los cuales los sistemas de gestión se constituyen en proyectos de gran éxito. Durante el año 2008, en Chile, se desarrolló un programa que se orientaba a la construcción de escenarios deportivos para el campeonato mundial de fútbol femenino sub20. En Perú, se construyó la Central Hidroeléctrica Platanal, reconociéndose como la empresa hidroeléctrica de mayor magnitud en este país. Por último, en Panamá se llevó a cabo un programa para expandir el comercio internacional, ampliando el Canal de Panamá. En común, los proyectos previamente enunciados se reconocen porque cuentan al interior de sus sistemas de gestión con el apoyo de estándares utilizados por el Project Management Institute (PMI), en aras de lograr el éxito en su ejecución. El PMI desarrolló la manera de gestionar cada proceso a tiempo, cómo monitorearlo y cómo seguirlo efectivamente (Buchtik, 2011).

Adicionalmente, tomando como referencia la relevancia que posee un sistema de gestión al interior de la realidad de las organizaciones, independientemente de su diseño organizacional, ciclo de vida, naturaleza o estilo de gestión, se pueden avizorar algunas conclusiones al respecto. En primer lugar, la literatura académica en el campo de la administración evidencia que aplicar los sistemas de gestión contribuye al mejoramiento de la capacidad organizacional, impactando en el crecimiento, la rentabilidad y la proyección del negocio, a la vez que se opone a los procesos de liquidación y cierre de las empresas, tras el establecimiento de prácticas de carácter empírico en las organizaciones. De igual manera, la aplicación de un sistema de gestión debe llevarse a cabo de forma mancomunada por todos los niveles jerárquicos de la empresa; es decir, su aplicación será de mayor contundencia en la medida en que se acompañe del desarrollo de prácticas de carácter participativo en la gestión del negocio, en el trabajo en equipo, la polivalencia, la distribución de utilidades y, sin duda alguna, un mejoramiento en el clima organizacional que se perpetúe a través del tiempo (Téllez, 2015). 


\section{METODOLOGÍA}

En el diseño metodológico que siguió esta investigación fue imprescindible aplicar el método de estudio de caso, buscando separar las partes que componen el problema identificado para analizarlas detalladamente, a partir del objeto de estudio. Es de resaltar que por ello se siguió primordialmente la metodología cuantitativa, sin dejar de lado elementos cualitativos en el estudio (Páramo, 2013). Por último, los pasos que se llevaron a cabo en la investigación fueron los siguientes:

Se contactó y visitó las diferentes unidades de análisis, de las cuales posteriormente se extrajeron los datos necesarios, con el fin de realizar un diagnóstico organizacional de la situación de la empresa. La intención de esta fase corresponde al conocimiento de los procesos que hacen parte del diseño organizacional, en aras de identificar los diferentes niveles jerárquicos y los roles que desarrolla cada persona al interior de la organización.

Es de resaltar que los datos recolectados tienen que ver con la gestión de la dirección de las viviendas, el control de las facilidades habitacionales, el uso de los recursos asignados, los procesos de cobranza, el incumplimiento normativo, el déficit de viviendas, el tipo de organización, la satisfacción de los clientes, el proceso de control de los trabajos asignados, las pérdidas en los recaudos y el clima laboral de sus colaboradores. A partir de lo anterior, el instrumento empleado fue la encuesta, que se componía de siete interrogantes, las cuales se aplicaron a la alta dirección y diversos colaboradores de la dirección de vivienda. Las preguntas fueron cerradas y se detallan a continuación:
1. ¿Cómo calificaría la actual administración de la entidad?
2. ¿Cómo calificaría el control de las viviendas?
3. ¿Cómo calificaría la administración de los recursos económicos?
4. ¿Cómo calificaría los actuales procesos administrativos?
5. ¿Cómo calificaría la aplicación de la normativa vigente?
6. ¿Cómo calificaría el servicio brindado en las oficinas?
7. ¿Cómo calificaría el clima laboral?

Luego de esta etapa, la investigación continuó con el análisis de datos derivados de las poblaciones muestrales, que en este caso representan, entre los clientes de la empresa objeto de estudio, un número de 80 sujetos; es decir, simbolizan el tamaño del universo (100 personas) con un porcentaje de confianza del $95 \%$ y el porcentaje correspondiente de error del $5 \%$. Para organizar los datos, se eligieron las categorías temáticas que tienen en común todas las investigaciones, para clasificar de esta manera dicha información. En esta fase se empleó el programa estadístico SPSS, tomando en cuenta los criterios de medición en las variables seleccionadas, mediante la estadística descriptiva y, específicamente, a partir del análisis de las medidas de tendencia central (Páramo, 2008).

Por último, teniendo en cuenta el diagnóstico identificado de la organización objeto de estudio, se procedió a evaluar todos los constructos que dan lugar a la elaboración teórica requerida, en aras de diseñar un modelo del sistema de gestión, como propuesta de solución al mismo. Sin olvidar que las variables a estudiar van en concordancia con el tipo de organización, los procesos de control, el clima y cultura organizacional entre otras. 


\section{RESULTADOS}

El objetivo de la investigación es analizar la aplicación de los principales factores inmersos en los sistemas de gestión en organizaciones de Colombia, teniendo en cuenta un ejercicio de estudio de caso aplicado al sector vivienda. Para esto, se llevó a cabo inicialmente un diagnóstico acerca de las fortalezas y debilidades de dicho sistema, obteniendo como valor promedio general 4,59 (bajo el estándar normal). En aras de triangular la información, se aplicó una encuesta a la muestra seleccionada, que se fundamentó en la administración de los recursos, el control de la vivienda, los procesos administrativos, la aplicación de la normatividad, la calidad del servicio y el clima laboral. A partir de las variables anteriores, se describen los principales resultados:

\begin{tabular}{|c|c|c|c|c|c|c|c|c|c|c|}
\hline \multirow[b]{2}{*}{ Opción } & \multicolumn{2}{|c|}{$\mathrm{N}$} & \multirow[b]{2}{*}{$\frac{.0}{\overline{0}}$} & \multirow{2}{*}{ 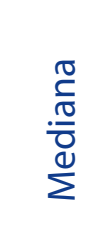 } & \multirow[b]{2}{*}{$\frac{\pi}{0}$} & \multirow{2}{*}{ 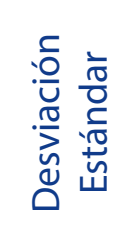 } & \multirow[b]{2}{*}{$\begin{array}{l}\frac{\mathbb{N}}{N} \\
\frac{0}{\frac{N}{2}} \\
\frac{\pi}{5}\end{array}$} & \multirow[b]{2}{*}{ 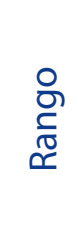 } & \multirow{2}{*}{$\stackrel{\stackrel{ }{E}}{\text { 를 }}$} & \multirow{2}{*}{ 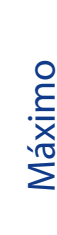 } \\
\hline & $\frac{\frac{0}{0}}{\sqrt{70}}$ & $\begin{array}{l}\tilde{o} \\
\frac{0}{0} \\
\overline{0} \\
\overline{0} \\
\alpha\end{array}$ & & & & & & & & \\
\hline $\begin{array}{l}\text { Como calificaría la actual } \\
\text { administración }\end{array}$ & 80 & 0 & 3,175 & 3,000 & 4,0 & 1,3574 & 1,842 & 4,0 & 1,0 & 5,0 \\
\hline $\begin{array}{l}\text { Como calificaría el control } \\
\text { de las viviendas }\end{array}$ & 80 & 0 & 3,013 & 3,000 & 2,0 & 1,3825 & 1,911 & 4,0 & 1,0 & 5,0 \\
\hline $\begin{array}{l}\text { Como calificaría la admi - } \\
\text { nistración de los recursos } \\
\text { económicos }\end{array}$ & 80 & 0 & 3,125 & 3,000 & 4,0 & 1,3722 & 1,883 & 4,0 & 1,0 & 5,0 \\
\hline $\begin{array}{l}\text { Como calificaría los actua - } \\
\text { les procesos administrativos }\end{array}$ & 80 & 0 & 2,988 & 3,000 & 2,0 & 1,4186 & 2,013 & 4,0 & 1,0 & 5,0 \\
\hline $\begin{array}{l}\text { Como calificaría la apli - } \\
\text { cación de la normativa } \\
\text { vigente }\end{array}$ & 80 & 0 & 3,050 & 3,000 & 5,0 & 1,4574 & 2,124 & 4,0 & 1,0 & 5,0 \\
\hline $\begin{array}{l}\text { Como calificaría el servicio } \\
\text { brindado en las oficinas }\end{array}$ & 80 & 0 & 2,925 & 3,000 & 3,0 & 1,3759 & 1,893 & 4,0 & 1,0 & 5,0 \\
\hline $\begin{array}{l}\text { Como calificaría el clima } \\
\text { laboral }\end{array}$ & 80 & 0 & 3,088 & 3,000 & 4,0 & 1,3235 & 1,752 & 4,0 & 1,0 & 5,0 \\
\hline
\end{tabular}

Tabla 1. Resultados de la encuesta realizada a la dirección de vivienda Fuente: Elaboración propia. 
Posteriormente, con los resultados obtenidos en las siete preguntas planteadas a la muestra, se procedió a la presentación de cada uno de éstos en forma detallada, para luego extraer las respectivas conclusiones.

Cabe mencionar, que la metodología de calificación de los parámetros en el instrumento aplicado se describe a profundidad en la Tabla 2.

\begin{tabular}{l|c}
\hline \multicolumn{1}{c}{ Modo de calificación para los parámetros de la encuesta } \\
\hline \multicolumn{1}{c}{ Categía } & Escala de medición \\
\hline Muy de acuerdo & 5 \\
\hline De acuerdo & 4 \\
\hline Neutro & 3 \\
\hline En desacuerdo & 2 \\
\hline Muy en desacuerdo & 1 \\
\hline
\end{tabular}

Tabla 2. Modo de calificación para los parámetros de la encuesta Fuente: Elaboración propia.

La primera pregunta de la investigación se circunscribió a la interpretación acerca de la gestión actual desarrollada en la empresa. El resultado obtenido, tal como se visualiza en la figura 3, es que un bajo porcentaje de tan solo el 35 \% tiene una percepción negativa. Ello coincide con el hecho de que 50 \% del personal otorga una calificación superior a 3,0, lo cual significa que el valor promedio en el que los participantes se ubican es 3,175. Además, los participantes que se desvían de dicho valor representan 1,3574 unidades de la escala.

La existencia de una amplia distribución entre los valores 1 y 5, es decir, muy en desacuerdo y muy de acuerdo, evidencia la necesidad de que los administradores de esta organización desarrollen prácticas en las cuales obtengan mayor realimentación de los diversos actores sociales y, de esta manera, mejorar las prácticas de gestión en la empresa.

En segundo lugar, se preguntó acerca de la calificación que se asigna al control de las viviendas. Ello evidenció que la variable de mayor repetición fue 2 , es decir, una calificación desfavorable. Los resultados denotan que la mayoría de la población prefiere asumir una posición neutral en relación con lo preguntado; en otras palabras, el 50 \% se encuentra se ubica por encima del valor 3,0 y la cifra restante debajo de este valor. Igualmente, en relación con el valor promedio en que se sitúan los participantes (3,013), se desvían 1,3825 unidades de la escala; ello significa una cifra semejante a la pregunta anterior. 
Cabe destacar que las respuestas obtenidas en relación con el control de las viviendas denotan que no existe homogeneidad en las posiciones acerca de ellas, lo cual apunta a debilidades en el proceso. En este tópico en particular, la aplicación de estrategias orientadas a mejorar este control es de carácter urgente, lo cual puede ir de la mano con el desarrollo del sistema de gestión propuesto.

La tercera pregunta se enfocó en calificar la administración de los recursos económicos de la organización objeto de estudio. Ello corresponde con las respuestas anteriores, donde las opiniones se encuentran divididas, a pesar de que la variable de mayor repetición fue 4. Por otro lado, los participantes continúan ubicándose en el valor neutral de 3,125 y solo el $34 \%$ asigna una calificación desfavorable.

Igualmente, se evidencia en los resultados que existe heterogeneidad en las respuestas otorgadas por parte de los encuestados. Sin duda alguna, esta situación pone de manifiesto la existencia de ciertas dificultades en el proceso de gestión de los recursos y denota fallas en el proceso de planeación de la empresa.

En cuar to lugar, se interrogó a la población seleccionada acerca de la calificación de los actuales procesos administrativos. Los resultados no denotan un cambio radical en las respuestas previas, a pesar de que debe señalarse la categoría 2 como la más repetida, es decir, que sobresale una opinión desfavorable en relación con la ejecución de estos procesos. A su vez, los participantes asignan un promedio un poco inferior $(2,988)$ a los interrogantes anteriores, con una desviación de 1,14186 unidades de la escala asignada.

A partir de lo anterior, se recomienda a la alta dirección de la empresa estudiada que efectúe una revisión de los procesos administrativos. Ello debe ir de la mano con la aplicación de un diagnóstico de mayor profundidad en cuanto a las percepciones de estos procesos, aclarando cuáles ajustes deben efectuarse en ellos.

La quinta pregunta efectuada buscaba conocer la calificación relacionada con la aplicación de la normativa vigente. En este sentido, se podría calificar de favorable la respuesta, ya que el parámetro de mayor repetición fue 5. También se da continuidad a los resultados previos en relación con la mediana y que denotan una percepción dividida en relación con la gestión de la organización. Así mismo, se desvían del promedio (3,050) un total de 1,4574 unidades.

Las respuestas a esta pregunta permiten inferir que la organización pudo tener mala publicidad en cuanto al cumplimiento de las leyes, aspecto que es de suma gravedad, ya que refleja falencias en la acción gerencial, así como en la aplicación del actual sistema de gestión. Igualmente, tal como en respuestas anteriores, se observa la existencia de heterogeneidad en los resultados, a partir de la presentación de datos ubicados entre 1 y 5 , es decir, muy de acuerdo y muy en desacuerdo.

En sexto lugar, se preguntó a los encuestados acerca de la calificación del servicio. En este caso, se manifiesta una valoración desfavorable, ya que, a pesar de repetir con mayor frecuenta el valor 3, los participantes se ubican en promedio en una opinión desfavorable (2,925). También se registró un desvío en promedio de 1,3759 unidades de la escala y el 20 \% de los encuestados presentó una opinión muy desfavorable en cuanto al servicio prestado.

La calidad del servicio se consolida como uno de los pilares en la satisfacción de las necesidades de los clientes. Por ende, la evaluación de los resultados enunciados, a pesar de que evidencia valores promedios y coherentes con las respuestas anteriores, muestra que la organización debe realizar mayores esfuerzos de capacitación a sus colaboradores para que a futuro ello no se traduzca en reducciones del segmento de mercado al que va dirigida la empresa.

La séptima pregunta se dirigió a la valoración en cuanto al clima laboral en la organización. La variable de mayor repetición fue favorable (4); además, se presenta un valor de la mediana equivalente a las respuestas previas. Del valor promedio (3,088), se desvían 1,3235 unidades y tan solo el $15 \%$ de los encuestados manifiestan una posición desfavorable en cuanto al clima laboral. 


\begin{tabular}{l|c|c|c|c} 
¿Cómo calificaría el clima laboral? \\
\multicolumn{1}{c|}{ Opción } & Frecuencia & Porcentaje & Porcentaje valido & $\begin{array}{c}\text { Porcentaje } \\
\text { acumulado }\end{array}$ \\
\hline Muy en desacuerdo & 12 & 15,0 & 15,0 & 15,0 \\
\hline En desacuerdo & 17 & 21,3 & 21,3 & 36,3 \\
\hline Neutro & 16 & 20,0 & 20,0 & 56,3 \\
\hline De acuerdo & 22 & 27,5 & 27,5 & 83,8 \\
\hline Muy de acuerdo & 13 & 16,3 & 16,3 & 100,0 \\
\hline Total & 80 & 100,0 & 100,00 & \\
\hline
\end{tabular}

Tabla 3. ¿Cómo calificaría el clima laboral? Fuente: Elaboración propia.

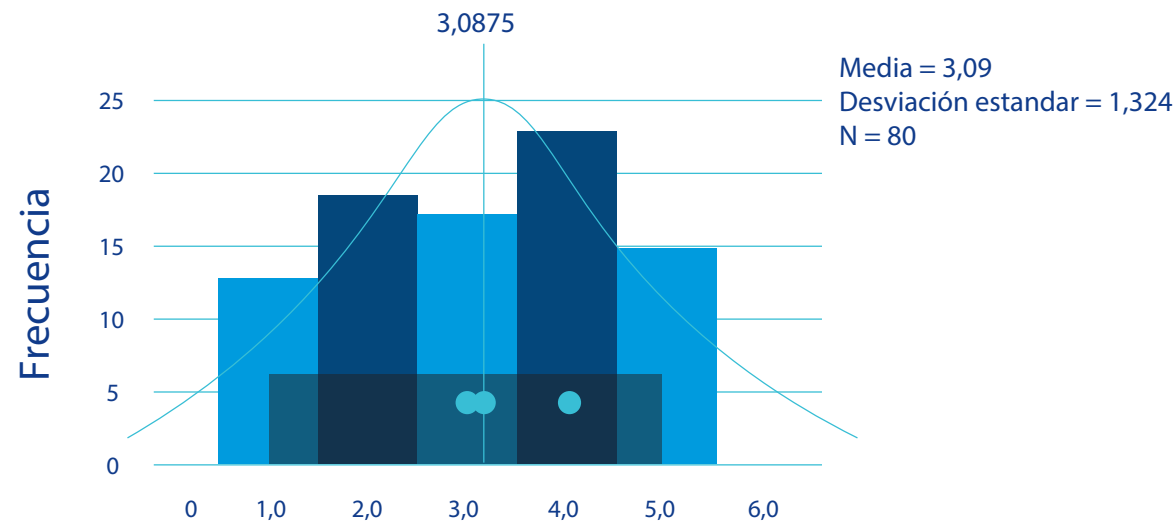

Figura 1. ¿Cómo calificaría el clima laboral? Fuente: Elaboración propia.

La distribución de los datos en esta pregunta muestra la heterogeneidad de opiniones acerca de este criterio de carácter interno de la organización. Igualmente, se requeriría contrastar estos datos con información que provenga de grupos focales o la aplicación de otro tipo de instrumentos de carácter cualitativo que facilite conocer a profundidad las razones acerca de este tipo de valoración y que además oriente a todos los colaboradores de la empresa. 


\section{DISCUSIÓN}

Los sistemas de gestión contribuyen al desarrollo y crecimiento de las organizaciones en los sectores público y privado, independientemente del sector económico, estilo de liderazgo, ciclo de vida o diseño organizacional. Por ende, urge en la actualidad que los administradores de las organizaciones desarrollen diagnósticos claros que identifiquen las fortalezas y limitaciones de las empresas, a la vez que promuevan cambios paulatinos en el mejoramiento de la gestión (Brunet, 2009).

La situación actual en la organización objeto de estudio permitió profundizar en relación con el tipo de organización, los procesos de control, el clima y la cultura organizacional, y que la proposición de estrategias y procesos que se recomienden sean incorporados en el diseño del sistema de gestión actual (Chiavenato, 2011; Robbins y Judge, 2009).

Por otro lado, la evaluación de los parámetros tomados en cuenta para el diseño del sistema de gestión en la dirección de vivienda, a través de las medidas de tendencia central (media, mediana y moda), ha conseguido determinar los aspectos en los cuales la empresa debe enfocar los esfuerzos y algunas de las medidas, como podría ser considerado el sistema de gestión (Koontz y Weihrich, 2012). Particularmente, en los resultados se socializa la necesidad de que la organización desarrolle acciones afirmativas para mejorar la percepción en cuanto al cumplimiento de la normativa y la calidad del servicio. Dichos elementos, de ser mejorados, incidirán en la percepción global de la gestión evaluada.

\section{CONCLUSIONES}

El objetivo general del presente artículo es analizar los factores que integran un sistema de gestión empresarial que permita optimizar la administración de los recursos humanos, materiales y financieros de los cuales las organizaciones disponen para el cumplimiento de sus actividades. En consecuencia, la principal conclusión es que los resultados arrojados en el estudio son poco alentadores; se evidencia que hoy no existe homogeneidad en las variables analizadas y ello manifiesta las falencias contenidas a lo interno de la empresa objeto de estudio.

Por otro lado, a medida que exista mayor aproximación y conocimiento de los requerimientos relacionados con el proyecto, con base en el diagnóstico y evaluación de los parámetros que forman parte del problema, mejor se comprenderán los beneficios que representan para la organización, al contar con un sistema de gestión efectivo para la administración de la vivienda. Por consiguiente, se recomienda a la organización solicitar los recursos económicos requeridos a la dirección general de finanzas, a fin de poder adquirir una plataforma informática de acuerdo con el diseño y necesidades planteados, entre otros, y que obedezcan al levantamiento de la información y a la problemática expuesta en el presente estudio. De igual forma, se sugiere socializar ante los clientes de la empresa analizada la implementación de la plataforma del sistema de gestión, con el propósito de minimizar el impacto por el cambio en la administración.

Para terminar, se identificó como principal limitación del estudio la selección de metodología cuantitativa, dada la necesidad de ampliar las características propias del objeto de estudio. En efecto, se recomienda para investigaciones posteriores desarrollar conjuntamente otros tipos de instrumentos de carácter cualitativo, los cuales faciliten el conocimiento de las causas en cuanto a las problemáticas identificadas. Igualmente, este trabajo representa un esfuerzo importante que podría ejecutarse en distintos sectores económicos, a la vez que facilitaría la gestión que actualmente desarrollan los empresarios en el país y que, sin lugar a dudas, teniendo en cuenta el surgimiento de algunas de estas organizaciones de manera espontánea y su gestión empírica, tienen mayores niveles de incertidumbre en el mercado. 


\section{REFERENCIAS}

Ahumada, P. (2001). La evaluación en una concepción de aprendizaje significativo. Ediciones Universitarias de Valparaíso.

Baena, G. (2015). Planeación prospectiva estratégica. Teorías, metodologías y buenas prácticas en América Latina. México: Universidad Nacional Autónoma de México.

Brunet, L. (2009). El clima de trabajo en las organizaciones: definiciones, diagnóstico y consecuencias. México: Trillas.

Buchtik, L. (2011). Proyectos exitosos en América Latina. Project Management Institute. Recuperado de http://americalatina.pmi.org/ /media/Files/latam/Argentina-Capitulo-Nuevo-Cuyo/2011-AR-NC-BuchtikProyectosExitosos.aspx

Chiavenato, I. (2011) Comportamiento organizacional: la dinámica del éxito en las organizaciones (2. ${ }^{a}$ ed.). México: Thomson.

Chiavenato, I. (2002). Introducción a la Teoría General de la Administración. México: McGraw-Hill.

Contreras, F. y Barbosa, D. (2013). Del liderazgo transaccional al liderazgo transformacional: Implicaciones para el cambio organizacional. Revista Virtual Universidad Católica del Norte, 2(39), 152-164.

Daft, R. (2007). Teoría y diseño organizacional. Cengage Learning Editores.

Fernández, A. (2005). Creatividad e innovación en empresas y organizaciones. Madrid: Díaz de Santos.

Fraguela, J., Carral, L., Iglesias, G., Castro, A. y Rodríguez, M. (2011). La integración de los sistemas de gestión. Necesidad de una nueva cultura empresarial. DYNA, 78(167), 44-49.

Franco, M. y Urbano, D. (2010). El éxito de las pymes en Colombia: un estudio de caso en el sector salud. Estudios Gerenciales, 26(114), 77-97.

Gómez, D., Rincón, H. y Niño, L. (2017). Retornos a la educación. Breve análisis comparativo de los salarios de los profesionales y ocupaciones entre 2010-2014 desde la perspectiva del capital humano. En Gómez, D. (ed.). Retail, Merchandasing y estudios de caso, 265-310. Bogotá, Colombia: Universidad de San Buenaventura.

Gómez, D. y Rincón, H. (2016). Perspectivas de cambio organizacional y aprendizaje en las organizaciones, una revisión de autores y teorías. Ponencia 51, Congreso Latinoamericano de Escuelas de Administración, CLADEA, ASCOLFA.

Inche, J., Chung P. y Salas, J. (2010). Diseño e implementación de un sistema de gestión de calidad académicoadministrativa en la Unidad de Posgrado de la Facultad de Ingeniería Industrial de la UNMSM. Industrial Data, 13(2), 9-14.

Losada, J., Bonilla, M. y Gómez, D. (2018). Gestión del talento humano en las pequeñas empresas colombianas: una aproximación bibliográfica. CreaE. Cuadernos de Emprendimiento, 3, 62-69.

Katz, D. y Kahn, R. (1978). The social psychology of organizations. New York: Wiley.

Koontz, H. y Weihrich, H. (2012). Administración: una perspectiva global y empresarial. México: McGraw-Hill.

Mintzberg, H. (1984). La estructura de las organizaciones. Barcelona: Ariel.

Nonaka, L., Takeuchi, H. y Umemoto, K. (1996). A theory of organizational knowledge creation. International Journal of Technology Management, 11(7), 833-845.

Páramo, P. (2013). La investigación en ciencias sociales: estrategias de investigación. Bogotá: Editorial Universidad Piloto de Colombia. 
Páramo, P. (2008). La Investigación en Ciencias Sociales: Técnicas de recolección de información. Bogotá: Editorial Universidad Piloto de Colombia.

Pardo, C. y Alfonso, W. (2015). Análisis de los principales factores del fracaso de los emprendimientos en Colombia. Instituto del Fracaso. Recuperado de https://thefailureinstitute.com/wp-content/uploads/2015/11/Fracasoemprendimiento-Colobia.pdf

Poole, M. y Van de Ven, A. (2004). Theories of organizational change and innovation processes. Handbook of organizational change and innovation. Oxford University Press. 374-397.

Porter, M. (1998). Clusters and the new economics of competition. Boston: Harvard Business Review.

Rincón, H., Niño, L. y Gómez, D. (2017). Retail en Colombia 2010-2015: un estudio a partir del análisis financiero integral como elementos de soporte de las tomas decisiones. Revista Espacios, 38(42).

Rengifo, A. y Ramírez, D. (2010). Diseño de un sistema de gestión administrativo para la asociación Asomujeres Proactivas en el municipio de Pereira Risaralda. Tesis de maestría. Universidad Tecnológica de Pereira, Colombia.

Schumpeter, P. (1967). Teoría del desarrollo económico. México: Fondo de Cultura Económica.

Robbins, S. y Judge, T. (2009). Comportamiento organizacional (13. ${ }^{\mathrm{a}}$ ed.). México: Pearson.

Téllez, C. (2015). Aproximación a la responsabilidad social empresarial hotelera en Colombia: reflexiones a partir de la perspectiva cualitativa. Tesis de maestría. Universidad Nacional de Colombia, Bogotá, Colombia. Recuperado de: http://www.bdigital.unal.edu.co/50805/1/1032366176.2015.pdf 